University of Nebraska - Lincoln

DigitalCommons@University of Nebraska - Lincoln

Roger Kirby Publications

Research Papers in Physics and Astronomy

May 1994

\title{
Temperature-dependent interface magnetism and magnetization reversal in Co/Pt multilayers
}

Z. S. Shan

Hangzhou University, Zhejiang, China

J. X. Shen

University of Nebraska - Lincoln

Roger D. Kirby

University of Nebraska-Lincoln, rkirby1@unl.edu

David J. Sellmyer

University of Nebraska-Lincoln, dsellmyer@unl.edu

Y. J. Wang

Chinese Academy of Sciences, Beijing, China

Follow this and additional works at: https://digitalcommons.unl.edu/physics_kirby

Part of the Physics Commons

Shan, Z. S.; Shen, J. X.; Kirby, Roger D.; Sellmyer, David J.; and Wang, Y. J., "Temperature-dependent interface magnetism and magnetization reversal in Co/Pt multilayers" (1994). Roger Kirby Publications. 18.

https://digitalcommons.unl.edu/physics_kirby/18

This Article is brought to you for free and open access by the Research Papers in Physics and Astronomy at DigitalCommons@University of Nebraska - Lincoln. It has been accepted for inclusion in Roger Kirby Publications by an authorized administrator of DigitalCommons@University of Nebraska - Lincoln. 


\title{
Temperature-dependent interface magnetism and magnetization reversal in $\mathrm{Co} / \mathrm{Pt}$ multilayers
}

\author{
Z. S. Shan, ${ }^{\text {a) }}$ J. X. Shen, R. D. Kirby, and D. J. Sellmyer \\ Behlen Laboratory of Physics and Center for Materials Research and Analysis, University of Nebraska, \\ Lincoln, Nebraska 68588-0111 \\ Y. J. Wang \\ Institute of Physics, Chinese Academy of Sciences, 100080 Beijing, China
}

\begin{abstract}
We report on the temperature dependence of the magnetic properties and interface magnetism of $\mathrm{Co} / \mathrm{Pt}$ multilayers. The magnetic properties including magnetization and anisotropy change substantially as the temperature varies from 300 to $10 \mathrm{~K}$ for samples with Co layer thickness in the range from 3 to $7 \AA$. The interface anisotropy of about $0.38 \mathrm{erg} / \mathrm{cm}^{2}$ is nearly independent of temperature. The magnetization reversal is dominated by domain wall motion for the thinner Co layers and dominated by nucleation for the thicker Co layers.
\end{abstract}

\section{INTRODUCTION}

$\mathrm{Co} / \mathrm{Pt}$ multilayers have attracted much attention as possible magneto-optical recording media because of their large perpendicular anisotropy, large Kerr rotation at short wavelength, and high corrosion resistance. ${ }^{1-5}$ However, aside from the study of magnetization by Bloemen et al. ${ }^{6}{ }^{6}$ very little work on the temperature dependence of the magnetic properties of this system has been presented. Recently, we have studied these properties and the magnetization reversal of $\mathrm{Co} / \mathrm{Pt}, \mathrm{Co} / \mathrm{Au}$, and $\mathrm{Co} / \mathrm{Pd}$ multilayers. In this paper we report our studies on $\mathrm{Co} / \mathrm{Pt}$, and the results for $\mathrm{Co} / \mathrm{Au}$ and $\mathrm{Co} / \mathrm{Pd}$ will be published elsewhere.

\section{EXPERIMENT}

$\mathrm{Co} / \mathrm{Pt}$ multilayers of the form $X \AA \mathrm{C}$ / $15 \AA \mathrm{Pt}(X=3,4,5$, $7,9,12,15$, and 20$)$ were fabricated by sputtering on Si(111) substrates with a $200 \AA$ Pt buffer layer. The sputtering rate and power for $\mathrm{Co}$ (DC gun) were $\sim 0.6 \AA / \mathrm{s}$ and $20 \mathrm{~W}$ and for Pt (RF gun) were $1.1 \AA / \mathrm{s}$ and $40 \mathrm{~W}$, respectively. The vacuum prior to sputtering was $1 \times 10^{-7}$ Torr and the Ar pressure during sputtering was $5 \times 10^{-3}$ Torr. All eight samples were prepared in one vacuum run to ensure the same preparation conditions.

Measurements of the hysteresis loops were carried out using an alternating gradient magnetometer (AGM) and SQUID from 300 to $10 \mathrm{~K}$. The measured magnetic anisotropy was determined from the area between the perpendicular and parallel (in-plane) magnetization curves. The time decay of Kerr rotation was measured on the apparatus described in Ref. 7. Structural properties were analyzed with both small and large angle $\mathrm{x}$-ray diffraction.

\section{STRUCTURE}

A small angle $x$-ray diffraction scan [see Fig. 1(a)] showed that the peaks corresponding to the multilayer structure appeared at the right positions and up to the fourth superlattice peak was observed for the thinnest Co layer sample $(X=3 \AA)$. Therefore all samples have distinct interfaces.

\footnotetext{
a'Permanent address: Dept. of Electronic Engineering, Hangzhou University, Hangzhou, Zhejiang, People's Republic of China.
}

Large angle $\mathrm{x}$-ray diffraction [see Fig. 1(b)] showed a pronounced FCC (111) texture, with the main peak falling between the pure $\mathrm{FCC} \mathrm{Co}(111)$ and $\mathrm{Pt}(111)$.

\section{MAGNETIC PROPERTIES}

Both perpendicular $\left(\mathbf{H}_{\perp}\right.$ film plane $)$ and parallel $\left(\mathbf{H}_{\|}\right.$film plane) hysteresis loops were measured from 300 to $10 \mathrm{~K}$ for all samples. The temperature dependences of magnetization, anisotropy, and polarization of $\mathrm{Pt}$ atoms are summarized as follows.

\section{A. Temperature dependences of magnetizations}

The temperature (or Co layer-thickness) dependences of saturation magnetization for $X=3,4,5,7,12$, and $20 \AA$ (or $T=10,100,200$, and $300 \mathrm{~K}$ ) are illustrated in Fig. 2(a) [or Fig. 2(b)]. The magnetization here is defined as the ratio of the measured moment to the Co mass, assuming that all the moment is from Co atoms. It is seen that (1) The samples with thinner Co layers show the stronger temperature dependence. As the temperature varies from 300 to $10 \mathrm{~K}$, the saturation magnetization increases by $30,20,13,6.7$, and $\sim 2.4 \%$ for $X=2,4,5,7$, and $12 \AA$, respectively. We notice that the $X=12$ and $20 \AA$ samples have essentially the same magneti-

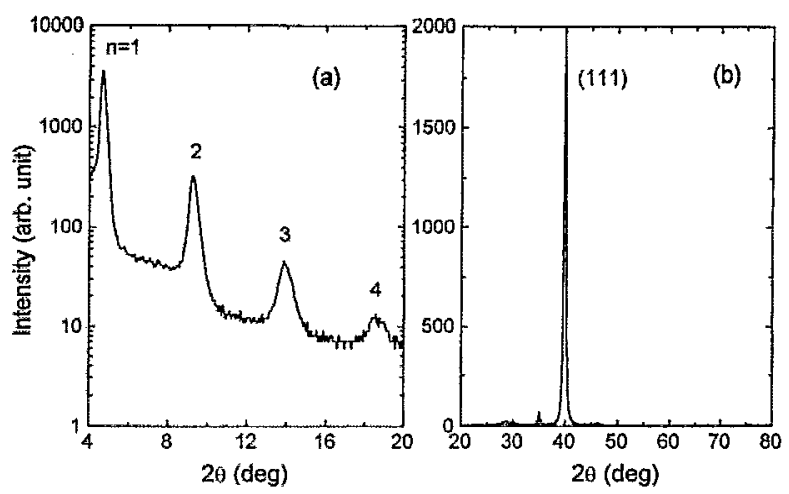

FIG. 1. CoK $K_{\text {ak }}$ (a) small-angle and (b) large-angle diffraction intensity for 3 $\AA \mathrm{Co} / 15 \AA \mathrm{APt}$ as a function of $2 \theta$. 

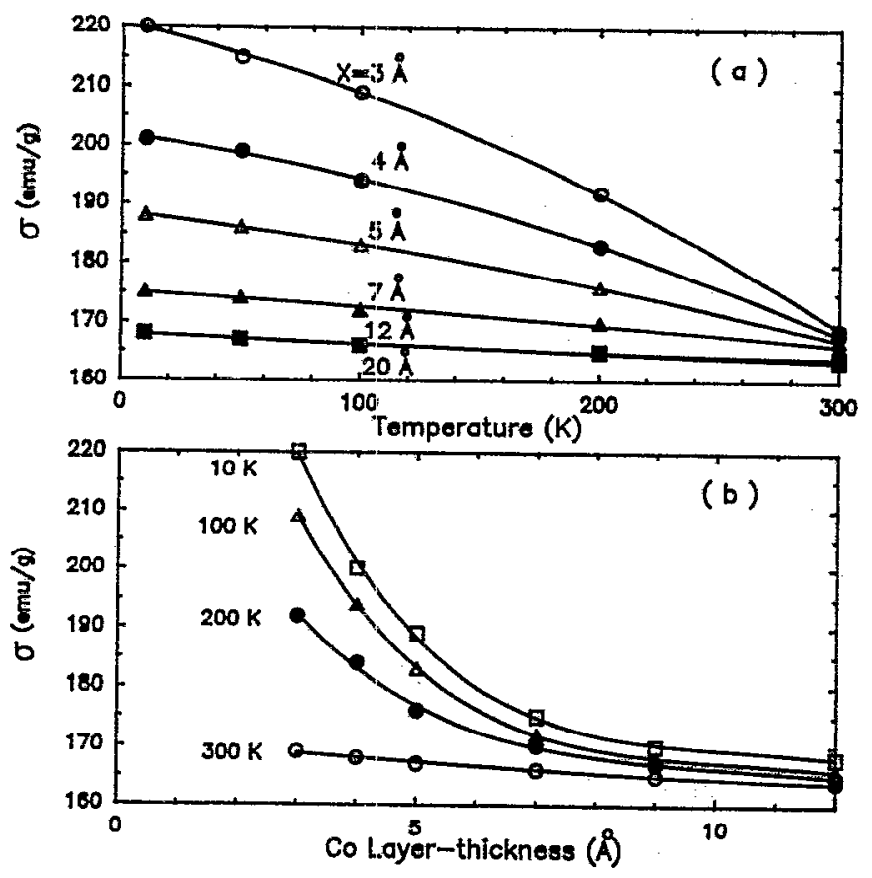

FIG. 2. Temperature dependence of $\mathrm{Co}$ saturation magnetization for (a) different Co layer-thickness and (b) layer-thickness dependence of Co saturation magnetization at different temperatures.

zations. (2) The Co layer-thickness dependence of magnetization becomes much stronger at the lower temperature $[$ see Fig. 2(b)].

It is well known that the magnetization of pure $\mathrm{Co}$ is only weakly temperature dependent ( $\sigma_{\mathrm{Co}}$ only increases $\sim 1$ 양 as the temperature decreases from 300 to $4.2 \mathrm{~K}$ ). The enhancement of magnetization in Figs. 2(a) and 2(b) thus originates from the multilayer structure and interface magnetism. Because of the interdiffusion or mixing between $\mathrm{Co}$ and $\mathrm{Pt}$ atoms at the interface region, as the Co layer becomes very thin, e.g., one to three atomic layers, the interfaces may approximately be regarded as a disordered $\mathrm{Co}-\mathrm{Pt}$ alloy. Then the polarization of $\mathrm{Pt}$ atoms and the temperature dependence of magnetization of the interfaces can be calculated in terms of the mean-field theory, as has been done for Co-Pd and $\mathrm{Co}-\mathrm{Cu}$ alloys in Refs. 8 and 9. We will not give a detailed discussion of the mean-field calculation to save space, and only point out the main result here: $\mathrm{Co}-\mathrm{Pt}$ alloys with smaller Co concentration have a lower ordering temperature and show a stronger temperature dependence of magnetization. Thus one might expect that thinner Co-layer samples, whose interfaces correspond to $\mathrm{Co}-\mathrm{Pt}$ alloys with smaller $\mathrm{Co}$ concentration, manifest the stronger temperature dependence of magnetization.

\section{B. Temperature dependences of anisotropy}

A summary of the temperature (or Co layer-thickness) dependencies of measured anisotropy for $X=3,4,5,7,12$, and $20 \hat{A}$ (or $T=10,100,200$, and $300 \mathrm{~K}$ ) are shown in Fig. 4(a) [or 4(b)]. It is worthwhile to mention the following: (1) The measured anisotropy $K_{u}$ is positive for $X \leqslant 9 \quad A$ and negative for $X \geqslant 10 \AA$ over the whole temperature range [see
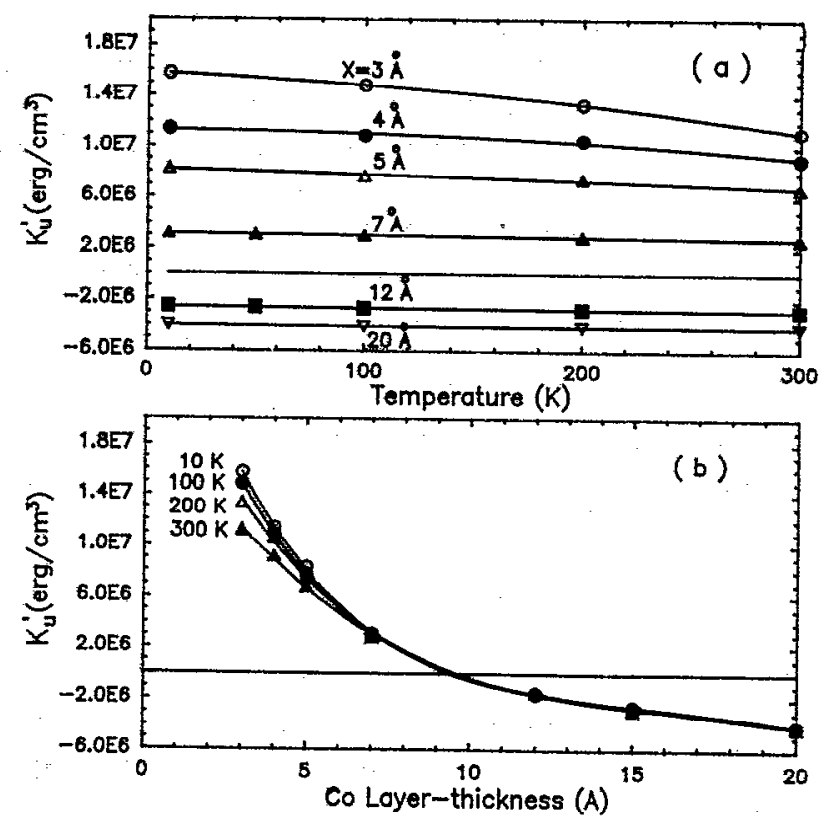

FIG. 3. Temperature dependence of measured anisotropy for (a) different $\mathrm{Co}$ layer thickness and (b) layer-thickness dependence of measured anisotropy at different temperatures.

Fig. 4(b)]. (2) Samples with thinner Co layers show a stronger temperature dependence of $K_{u}$ [see Fig, 4(a) and 4(b)]. $K_{u}$ values increase by $40 \%, 24 \%$, and $22 \%$ for $X=3,4$, and $5 \AA$ as temperature decreases from 300 to $10 \mathrm{~K}$. (3) We have determined the interface anisotropy $K_{i}$ for these samples using the standard method of plotting " $\lambda K_{u}$ vs $d_{\mathrm{Co},}$ " as described in Refs. 9 and 10 . We found $K_{i}$ to be $\sim 0.38$ $\left(\mathrm{erg} / \mathrm{cm}^{2}\right) . K_{i}$ is also nearly independent of temperature. The fact that the samples with thinner Co layers $(X=3,4$, and 5 $\AA$ ) show stronger temperature dependences of the anisotropy, while, for samples with $X \geqslant 7 \AA$ the anisotropy is nearly independent of temperature, implies that the interfaces give the major contribution to the temperature dependence the of anisotropy.

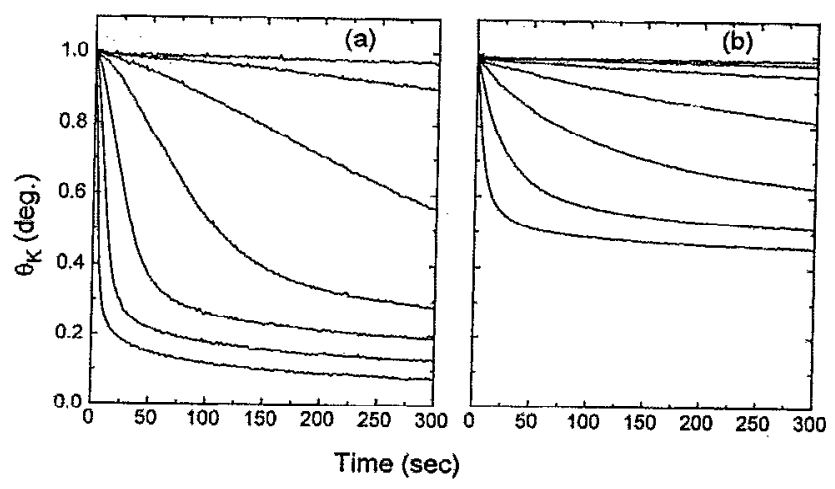

FIG. 4. Kerr rotation as a function of time after magnetization reversal for (a) $3 \AA \mathrm{Co} / 15 \AA \mathrm{Pt}$ and (b) $5 \AA \mathrm{Co} / 15 \AA \AA \mathrm{Pt}$. Different curves correspond to the different reversing fields ( $\leqslant H_{c}$ ), with the uppermost curve corresponding to the smallest field. 
To study the origins of anisotropy, $\mathrm{Co} / \mathrm{Pt}$, and $\mathrm{Co} / \mathrm{Au}$ multilayers were prepared, and their temperature dependencies of the anisotropy $K_{u}$ have been measured. We note that the thermal expansion coefficients of $\mathrm{Au}, \mathrm{Co}$, and $\mathrm{Pt}$ are $14.2 \times 10^{-6}, 12 \times 10^{-6}$, and $9 \times 10^{-6}\left({ }^{\circ} \mathrm{C}^{-1}\right)$, respectively. ${ }^{11}$ Also, the expansion coefficient of $\mathrm{Au}$ is greater than that of $\mathrm{Co}$, while the expansion coefficient of $\mathrm{Pt}$ is less than that of Co. Therefore, if stress anisotropy were the major source of $K_{u}$, one would expect that the anisotropy of $\mathrm{Co} / \mathrm{Pt}$ and $\mathrm{Co} / \mathrm{Au}$ would show opposite temperature dependences: one increasing with decreasing temperature while the other decreasing with decreasing temperature. However, our experiment shows that both for $\mathrm{Co} / \mathrm{Pt}$ and $\mathrm{Co} / \mathrm{Au}$ multilayers, the $K_{u}$ values increase with decreasing temperature for samples with thin Co layers. This may be regarded as evidence that stress plus inverse magnetostriction is a minor contribution to the anisotropy in our (111) textured samples, in comparison with magnetocrystalline anisotropy generated by spinorbit interaction in the highly anisotropic multilayers structure. ${ }^{12,13}$

\section{Magnetization reversal}

Magnetization reversal can be studied by the time decay of Kerr rotation. The measurement procedure was described in Ref. 7, and results are given in Figs. 5(a) and 5(b) for $X=3$ and $5 \AA$, respectively.

Several authors ${ }^{14,15}$ have pointed out that if the nucleation rate is small and the domain wall motion velocity is large, the decay curve will first decrease slowly by nucleation at a few isolated sites and then fall quickly through the rapid expansion of the domain walls. The decay curves in Fig. 5(a) are consistent with this behavior. On the other hand, if the nucleation rate is large and the domain wall velocity is very small, the decay curve first decreases exponentially and then decreases approximately as $\ln (t)$ ( $t$ is the time) at long times. The decay curves in Fig. 5(b) show such behavior. The $\ln (t)$ behavior can arise either as a consequence of a distribulion of thermal activation energics or because part of the driving force (the demagnetizing field) for reversal is decreasing with time as the sample reverses. ${ }^{16}$ Therefore the reversal is dominated by domain wall motion for samples with thinner Co layers, while it is dominated by nucleation for thicker $\mathrm{Co}$ layer samples. Presumably this difference in behavior results from details of the nanostructures (defects, interface mixing, etc.), as well as differences in demagnetizing fields, both of which must be further investigated to obtain a clear understanding of the reversal behavior.

\section{CONCLUSIONS}

It is concluded that the substantial temperature dependence of the magnetic properties of the thin Co layer multilayers originates from the alloying effect at the interfaces. As the temperature decreases, the enhancement of magnetization can be understood in terms of mean-field theory and the enhancement of anisotropy results from the interfacial magnetism, however, the detailed mechanism still remains to be solved. This is a challenging problem when interfacial mixing is present, because up to the present time the theoretical calculations of magnetic anisotropy in multilayers have assumed perfect boundaries with no atomic disorder. Some experiments were performed to probe the origin of the anisotropy. It was found that stress plus magnetostriction does not appear to play a dominant role. Magnetization reversal is dominated by wall motion and nucleation for thinner and thicker Co layers, respectively.

\section{ACKNOWLEDGMENTS}

This work was supported by the National Science Foundation under Grant No. DMR-9222976, for which we are grateful. The authors would like to thank S. S. Jaswal and A. Runge for assistance and helpful discussion.

'W. B. Zeper, H. W. van Kesteren, B. A. J. Jacobs, J. H. M. Spruit, and R. F. Carcia, J. Appl. Phys. 70, 2264 (1991).

${ }^{2}$ S. Hashimoto, Y. Ochiai, and K. Aso, J. Appl. Phys. 67, 2136 (1990).

${ }^{3}$ F. J. A. den Broeder, W. Hoving, and P. J. H. Bloemen, J. Magn. Magn. Mat. 93, 562 (1991).

${ }^{4}$ T. Suzuki, H. Notarys, D. C. Dobbertin, C. J, Lin, D. Weller, D. C. Miller, and G. Gorman, IEEE Trans. MAG-28, 2754 (1992).

${ }^{5}$ Y. J. Wang and W. Kleemann, J. Magn. Magn. Mat. 115, 9 (1992).

${ }^{6}$ P. J. H. Bloemen, W. J. M. de Jonge, and F. J. A. den Broeder, J. Magn. Magn. Mat. 93, 105 (1991)

${ }^{7}$ J. X. Shen, R. D. Kirby, and D. J. Sellmyer, J. Magn. Magn. Mat. 81, 107 (1989).

${ }^{8}$ Z. S. Shen, P. He, C. Moore, J. Woollam, and J. Sellmyer, J. Appl. Phys. 73, 6057 (1993).

${ }^{9}$ Z. S. Shen, S. Nafis, J. Woollam, S. H. Liou, and J. Sellmyer, J. Appl. Phys. 73, 6347 (1993).

${ }^{10}$ P. F. Carcia, Appl. Phys. Lett. 47, 178 (1985).

${ }^{11}$ Handbook of Chemistry and Physics 52 nd ed., edited by R. C. Weast (Chemical Rubber, 1971-1972), p. D-141.

12 J. M. Maclaren and R. H. Victora, IEEE Trans. Magn. MAG-24, 3034 (1993).

${ }^{13}$ Daalderop, P. J. Kelly, and M. F. H. Schuurmans, Phys. Rev. B 42, 7270 (1990).

${ }^{14}$ M. Labrune, S. Andrieu, F. Rio, and P. Bernstein, J. Magn. Magn. Mat. 80, 211 (1989)

${ }^{15}$ R. D. Kirby, J. X. Shen, R. J. Hardy, and D. J. Sellmyer (unpublished).

${ }^{16}$ D. K. Lottis, R. White, and E. D. Dahlberg, Phys Rev. Lett. 67, 362 (1991). 